\title{
Patrones de isoglosas en el español de Chile
}

\section{Patterns of isoglosses in the Spanish language of Chile}

\author{
CLAUDIO WAGNER \\ Academia Chilena de la Lengua
}

Con materiales procedentes del Atlas Lingüístico y Etnográfico de Chile por regiones, ALECh, nos proponemos determinar los patrones de isoglosas que permitirían establecer zonas dialectales en el español hablado de Chile, y relacionarlos con las fronteras lingüísticas propuestas como hipótesis para determinar cuatro "regiones" o zonas dialectales en el español de Chile, identificando de paso centros irradiadores y probables causas de las innovaciones detectadas.

Palabras clave: variaciones dialectales, atlas lingüístico, zonas dialectales, patrones de isoglosas.

Taking information from the Atlas lingüístico y etnográfico de Chile by regions, ALECh, we intend to determine the patterns of isoglosses that would allow to establish dialect areas in the Spanish language of Chile, and to relate them to the linguistic borders proposed as hypotheses to determine four "regions" or dialect zones in the Spanish of Chile, identifying in passing radiating centers and probable causes of the registered innovations.

Key words: dialect variations, linguistic atlas, dialect zones, patterns of isoglosses. 
Considerando que el lenguaje de un pueblo constituye el patrimonio cultural inmaterial más profundo y que mejor lo caracteriza, un atlas lingüístico se constituye en un instrumento insustituible de diagnóstico lingüístico global de un territorio determinado, perdurable por mucho tiempo, dada la lentitud natural de los cambios que se producen en la lengua. Un análisis trascendente de los materiales lingüísticos recolectados revelará que es uno de los pocos instrumentos que puede enseñarnos mucho acerca de una serie de realidades no lingüísticas, como la influencia de inmigraciones sobre la propia lengua, la relación entre límites políticos y fronteras lingüísticas, la existencia de pueblos originarios hoy desaparecidos, y otras, mientras que un análisis inmanente, gracias a la metodología utilizada y a la distribución espacial de esos materiales, puede permitirnos construir hipótesis mínimamente justificables sobre zonas dialectales en un territorio o dominio determinado, cuestión que ahora nos interesa.

Aunque las variaciones dialectales se dan en cualquier nivel de la lengua, el léxico es sin duda el más atractivo por ser el que está referido más estrechamente a la cultura de un pueblo, lo que explica que no pocas veces sea el único testimonio de la existencia de un objeto, costumbre o acontecimiento hoy ya desaparecidos.

Los estudios de geografía lingüística o geolingüística confirman un fenómeno interesante respecto del léxico, y es que cada palabra tiene su historia, lo que se ve con claridad, por ejemplo, con los préstamos lingüísticos. Pero si cada palabra tiene su propia historia, los procesos a que están sometidas son contados y pueden afectar a más de una, como ocurre, entre otros, con la adopción de una palabra por otra lengua o dialecto y su eventual adaptación posterior; con la desaparición de una palabra y su remplazo por una forma innovadora, hecho precedido normalmente por una pugna, de duración indeterminada, entre la forma arcaizante y la innovadora; con el intercambio de palabras entre variedades internas -geográficas, socioculturales, situacionales, generacionales- de una lengua común; con la sobrevivencia de la forma arcaizante, como residuo léxico, en una o más áreas marginales de un territorio; con las colisiones homonimicas por confluencia evolutiva de dos vocablos, lo que obliga a su diferenciación.

Y lo interesante es descubrir que todos estos procesos de cambio obedecen siempre a necesidades expresivas de los usuarios, como ya lo planteara Coseriu.

La geolingüística también ha demostrado que las diferencias dialectales son graduales y no abruptas, por las necesidades de ajuste comunicativo entre los usuarios. La experiencia en países europeos, con una larga historia, como Alemania e Italia, por ejemplo, demuestra que solo entre un extremo y otro del territorio se pueden comprobar diferencias dialectales abruptas, no así en áreas contiguas, donde habitualmente impera la transición, donde las diferencias son necesariamente progresivas, por lo que el trazado de una isoglosa es teóricamente discutible, en razón de que ella supone una variación brusca desde el punto de vista geográfico.

Sin embargo, en ocasiones ocurre que las características de una barrera, natural, cultural o étnica, son tan marcadas que un número significativo de isoglosas tiende a agolparse en torno a ella -aunque rara vez son contiguas- como ocurre con los haces de 
isoglosas más famosos que han sido postulados: el haz de isoglosas conocido como La SpeziaRimini, que separa las variedades noritalianas de las centrales de Italia (Rohlfs 1952: 47 ss.) y el de Ancona-Cerros Albanos, que separa las variedades centrales de las meridionales, configurando lo que Rohlfs denomina "las tres Italias" (V. mapa 1).

No sucede lo mismo con el conjunto de isoglosas que se conoce como línea de Benrath, que corre de este a oeste a través de Alemania y Holanda en una línea ligeramente al norte de Berlín y separa el bajo alemán del alto alemán antes de terminar en el llamado abanico del Rin (Bloomfield 1964: 417), donde la distinción aludida ya no es posible, porque las isoglosas que representan los diferentes rasgos toman caminos separados, por lo que resulta prácticamente imposible establecer alguna generalización sobre el bajo y alto alemán en el área del Rin (V. mapa 2).

Aunque la incompatibilidad entre los conceptos de variación de habla e isoglosa de que se hablaba más arriba, es corroborada en la práctica por la existencia habitual de zonas de transición entre dos áreas contiguas que difieren en algún rasgo lingüístico, la noción de isoglosa -y por ende, de haz de isoglosas- resulta de todas maneras una "herramienta" de utilidad, aunque sea limitada, en la descripción del habla en una zona de transición. En efecto, se podría identificar esta con la doble isoglosa o heteroglosa, por ejemplo, para precisarla con posterioridad, y en un territorio con áreas no contiguas ya muestra su utilidad, puesto que la delimitación de estas es posible precisamente con una isoglosa específica (V. más adelante "patrón de áreas residuales"), sin contar con que "a veces las isoglosas se correlacionan con otros aspectos de la cultura regional cuya distribución se puede trazar geográficamente" (Chambers y Trudgill (1994: 158-61 y 165, 66).

En lo que respecta a las diferencias dialectales en el español, el establecimiento de áreas dialectales es uno de los temas de dialectología hispánica en los que menos se ha avanzado y, en lo que respecta al español de América, aunque se han hecho diversas propuestas, los materiales sobre los que se han realizado han sido parciales, escasos y de procedencia metodológica diversa y de rigor inconstante, al decir de Moreno Fernández (1993:13).

Como estos instrumentos no abundan precisamente para América ${ }^{1}$, las dificultades que han tenido los investigadores que han querido elaborar una descripción del español hablado en América ajustada a los hechos, se han debido, en su mayor parte, a la falta de materiales de primera fuente, por lo que se han debido conformar con datos escasos y heterogéneos.

Porque está claro que sin el conocimiento de la distribución geográfica de los fenómenos no se puede siquiera pretender postular las isoglosas que hipotéticamente separen dos áreas dialectales. Menos aún, pretender determinar si nos encontramos o no ante dos dialectos, ya que la confluencia de usos lingüísticos en un área determinada necesita ser

\footnotetext{
1 De los atlas lingüísticos americanos, varios están en curso, como el ADDU, de Uruguay y el ALiB de Brasil, otros desgraciadamente han quedado en el camino, y solamente hay tres concluidos: el ALEC (1982), el ALMex (1990-2003) y el ALECh, recientemente editado en su versión digital (2016).
} 
demostrada por medio de estudios geolingüísticos, y sociolingüísticos, agregan otros (cf. Moreno Fernández 1993: 16).

De esto parecen estar más o menos conscientes los investigadores que se han arriesgado a elaborar propuestas de división del español de América, por lo que en lugar de hablar de dialectos hablan de áreas o zonas dialectales, solución más aparente que real, ya que soslayan un punto de doctrina esencial: las áreas dialectales tanto como los dialectos se determinan a posteriori, esto es, con posterioridad al examen de los hechos lingüísticos registrados, y no antes de siquiera tenerlos.

Ahora bien, la dificultad de establecer zonas o áreas dialectales en América es la dificultad de establecer isoglosas que las separen, cuestión que se ha intentado explicar de varias maneras. Winkelmann (1996: 342-43) escribe:

En la Nueva Romania, es decir, en regiones lingüísticas de colonización no existen fronteras lingüísticas bien definidas, ya que no se dieron las condiciones necesarias para su formación, especialmente el aislamiento relativo de un dominio lingüístico durante un largo periodo de tiempo. Así, por ejemplo, en el Atlas lingüístico-etnográfico de Colombia (ALEC) difícilmente se encontrará una fragmentación dialectal tan fuerte como la que aparece en Asturias.

Esto significa que las áreas o regiones de colonización corresponden en general a asentamientos más recientes, con una diferenciación dialectal menos marcada que los asentamientos prolongados o más antiguos de los territorios de origen de los colonos.

Pero también significa que si la colonización se efectúa en etapas, los rasgos dialectales bien diferenciados de los asentamientos más antiguos "tienden a compartirse en distancias relativamente grandes, cuando el asentamiento se remonta a tan solo uno o dos siglos", según Chambers y Trudgill (1994:145). Dicho de otra forma, las isoglosas que identifican áreas dialectales en un territorio dado, como por ejemplo, el de los estados atlánticos norteamericanos, tienden a desvanecerse y hasta a desaparecer en territorios de colonización más reciente, como los del interior de dicho país, el medio Oeste y California.

Canfield, por su parte, en 1988 intentaba explicar otra situación corriente en América. Decía:

Zonas de América que distaban entre sí miles de kilómetros se colonizaron simultáneamente y recibieron idéntico estadio de lengua y cultura hispánicas a través de los mismos puertos del sur de España. Por esta razón, no se encuentran en América netas isoglosas correspondientes a rasgos estructurales, y el intento de delimitar áreas lingüísticas en dicho continente produciría un dibujo semejante a la piel de leopardo (cit. Pottier 1992: 208-209).

El examen del Atlas lingüístico de México, ALMex (Wagner 2003: 191), del Atlas lingüístico-etnográfico del sur de Chile (ALESUCh) (Araya 1868) y de los materiales ya procesados del ALECh (Wagner 2016) puede respaldar efectivamente esa afirmación. No obstante, con cierta dificultad también se pueden comprobar diferencias léxicas, fónicas y 
hasta gramaticales entre las localidades, pero la diferenciación dialectal no se da normalmente por la presencia de una variante $a$ en un área, y de una variante $b$-o su ausencia- en otra, sino en la mayor o menor frecuencia del rasgo lingüístico presente en ambas áreas, y es esa diferencia de frecuencia la que permitiría caracterizar -débilmente, es verdad- unas áreas dialectales frente a otras. La imagen de Canfield de un mapa cuya distribución de datos se asemejaría a la piel de leopardo parece ajustarse a la realidad, según se podrá ver más adelante.

El descrito es, por cierto, el patrón de isoglosas habitual con el que nos hemos encontrado al examinar el material del ALECh,: isoglosas que se entrecruzan y van en distintas direcciones al punto que pudiera hablarse de una ausencia de patrón, con el resultado de la imposibilidad de delinear muchas veces áreas bien definidas y, consecuentemente, de establecer isoglosas, simplemente porque la distribución de las unidades no logra conformar áreas homogéneas que no estén permeadas por otras unidades distintas. Una isoglosa en estas circunstancias se hace casi imposible, porque no define un área frente a otra, ya que la unidad lingüística aparece distribuida por todo el territorio, en manchas más o menos grandes rodeadas de otras que representan a unidades diferentes. Este patrón puede ser denominado, siguiendo a Canfield, "patrón de piel de leopardo".

Es lo que se observa, por ejemplo en los materiales que analizamos como muestra (V. mapa 3). El mapa que pregunta por <pasamanos > entrega como respuesta dos vocablos que predominan abrumadoramente en todo el territorio sobre otras tres o cuatro variantes menores que aparecen en una o dos ocasiones cada una. Ellos son baranda, con 151 ocurrencias, y pasamanos, con 71. La relación entre estas variantes mayoritarias es muy regular, puesto que la primera se impone en las cuatro zonas prácticamente doblando a la segunda, y su distribución recuerda la piel de leopardo de que habla Canfield. En efecto, ambos vocablos se entremezclan, unas veces agrupados en núcleos menores homogéneos, otras, formando islotes en medio de una mancha donde predomina la otra variante.

Lo mismo ocurre con las respuestas al concepto <la rayuela> (V. mapa 4), que revelan una pugna entre rayuela y tejo, que en la actualidad es ampliamente favorable para la primera variante, que dobla, en términos absolutos y casi matemáticamente, a la segunda (184 ocurrencias frente a 91 respectivamente). El análisis se torna incluso más interesante cuando se hace al interior de cada una de las cuatro macrorregiones que hemos postulado como hipótesis de probables zonas dialectales y también con propósitos prácticos de recopilación de datos para el ALECh².

En efecto, tratándose de un juego tradicional, no es extraño que la región o zona central, con Santiago como el centro de gravedad más influyente del país, retenga mayoritariamente el primer término (65 ocurrencias) con clara ventaja sobre el segundo (que presenta sólo 14) y se haya irradiado hacia las otras regiones, especialmente hacia la

\footnotetext{
2 Esas cuatro macrorregiones serían: región norte, desde el paralelo o grado 17, que se separa de la central por el Cordón de Chacabuco, paralelo 32; región central, separada de la región sur por la línea que establecen los ríos Itata-Nuble, paralelo 36,5; y región sur, separada de la región sur-austral por la línea Mehuincerros Huiple y Tralcán-Panguipulli, paralelo 39,5 hasta el 54.
} 
región norte, donde tejo prácticamente se desconoce. No ocurre lo mismo con la región sur, donde rayuela y tejo están a corta distancia (46 frente a 32), y sobre todo, con la región sur-austral, donde se invierte la relación, ya que tejo se impone sobre rayuela, doblándolo en número de ocurrencias ( 42 frente a 20). El entrecruzamiento de las variantes de nuevo dificulta enormemente el trazado de isoglosas.

Un tercer ejemplo: entre los nombres que responden a <la hoja de la ventana> (V. mapa 5), dos son los vocablos que se presentan a lo largo de todo el territorio: hoja, que predomina en las cuatro regiones, con 127 ocurrencias, seguido por ventana, con 48, respuesta esta última que en rigor presupone la indistinción del objeto por cuyo nombre se indaga. El panorama se hace más complejo con la presencia de una tercera forma, recogida en la región norte, y desconocida en el resto del país: ala, con 7 ocurrencias, y una cuarta, mano, que es inexistente en la región austral y casi inexistente en las regiones norte y central (4 y 5 ocurrencias respectivamente), pero que en la región sur, con 17 ocurrencias, compite con hoja, y toma el lugar que ventana tiene en las demás regiones.

Otro patrón no tan frecuente de isoglosas -que también recogen Chambers y Trudgill (145)- tiene que ver con una isoglosa específica que delimita áreas no contiguas en dos o más lugares del territorio encuestado. Dicho de otra forma, un rasgo lingüístico aparece en dos o más áreas del territorio, pero ellas están separadas por otra área en la que se da un rasgo diferente. Este patrón es indicio de que el rasgo que ahora aparece en áreas laterales se daba antes también en la intermedia, evidenciando así diferencias diacrónicas entre el rasgo extendido anteriormente en el territorio y la innovación (que puede ser más de una) que irrumpió en dicho territorio, fragmentándolo. Ese rasgo es ahora un rasgo residual, y el patrón que repite es la antítesis del anterior en la medida en que muestra un rasgo concentrado en más de un área, por lo que puede ser denominado "patrón de áreas residuales".

Un buen ejemplo es el que muestra el mapa dedicado al <hijo menor $>$ (V. mapa 6). Este revela que concho es la variante predominante de norte a sur del territorio $(62 \%$ del total de respuestas). La variante el menor también se recoge en todo el territorio, pero con una diferencia de frecuencia notable de apenas un 5\%, y siempre se registra junto con otras palabras, generalmente con concho. Por aparecer concentrada, además, en la zona central del país, no parece aventurado interpretar el menor como la variante correspondiente al estrato español más antiguo, sustituida en época posterior, sin data precisa, por el neologismo concho, expresión metafórica en el sentido de 'lo que queda, lo que resta', exitosa sin duda por su enorme expresividad. El examen de los materiales revela varios hechos interesantes, pero ahora nos interesa destacar la existencia de un área continua entre $29^{\circ}$ y $32^{\circ}$ del territorio -que parece ser el núcleo del fenómeno-, área dominada por las variantes pucho y puchusco, que reaparecen más al norte, en dos islotes, y más al sur, ya en la región central, en otros dos puntos aislados.

Las dos variantes pueden ser consideradas como una sola, porque, aunque se nos muestran como palabras diferentes, parecen tener un mismo lexema de base, lo que el estudio etimológico confirma: pucho tiene su origen en el quechua "puchu", que se conoce 
en Chile a través del mapudungun, que tomó incluso el verbo quechua "puchuy" 'sobrar', en tanto que puchusco sería la forma del participio del mismo verbo: 'lo que ha sobrado' (Lenz [1987]: \# 1167 y Supl. I).

Lo que sorprende inicialmente es que en la región sur-austral del territorio nos encontramos con otra área continua, coincidente con el archipiélago de Chiloé, entre $42^{\circ}$ y $43^{\circ}$, y cinco islotes aledaños a esta área ${ }^{3}$, donde la respuesta a $<$ hijo menor> es quepucho (con sus variantes fónicas capucho y copucho).

El estudio etimológico realizado por Lenz establece una relación entre esta variante y la variante nortina que acabamos de examinar, porque tendrían el mismo origen quechua. Esto significa que pucho y puchusco en el norte, y quepucho en el sur, se nos revelan actualmente como áreas discontinuas que constituyen un residuo del estrato anterior conformado por el préstamo quechua incorporado al español por mediación del mapudungun y extendido por un vasto territorio. Aparecen hoy como áreas laterales respecto de un área central cubierta por nuevos neologismos, especialmente por concho, que habría predominado sobre los otros por su enorme expresividad, como se ha dicho, hipótesis que permitiría asimismo explicar las variantes de pucho mencionadas, que es más plausible se hayan producido en la primera lengua receptora, el mapudungun, y no en la segunda, el español. El patrón de dichas áreas residuales es fácilmente reconocible.

El ejemplo que sigue tiene algunas similitudes con el anterior. Se trata del concepto $<$ brote de la papa almacenada $>$ (V. mapa 7), que es expresado por brote en todo el país, pero en la región norte tiene carácter exclusivo, evidenciando un primer estrato léxico. En tres localidades meridionales de la región central, nos encontramos con la variante tallo, que se extiende hacia el sur, llegando hasta Porvenir, en Tierra del Fuego; pero a partir del grado 38 en la región sur, en la Araucanía, se encuentra la voz de origen mapuche troyo dispersa en tres pequeñas áreas ( 5 localidades), contendiendo con brote y tallo, pero también existe en una localidad fronteriza de la región sur-austral.

Más al sur, muy cerca de la línea Mehuín-Panguipulli, frontera que separa-según nuestra hipótesis- la región sur de la sur-austral-, al brote de la papa almacenada se le llama choyo, palabra usada en un área más amplia que troyo (15 ocurrencias), bien delimitada y exclusiva.

Ahora bien, según Lenz (Dicc.: \#\# 810, 1561), choyo es palabra de origen mapuche, pero nada dice de troyo, que tampoco aparece en otros diccionarios. Sin embargo, como no es extraña en mapudungun la sustitución entre los sonidos alveolar africado [t $\mathrm{t}^{\mathrm{r}}$ y palatal africado [ch] (cp. Catrileo 2010: 48) a través de alguno de sus varios alófonos por la cercanía de posición, en ejemplos como trotroca - chochoca 'especie de milcao', tranque - chanque 'amigo, casero' o trile - chili 'cierto pájaro negro con mancha amarilla en las alas' (Lenz: \#\# 1398, 1376, 1384), podemos sostener que las voces que nos interesan son variantes

\footnotetext{
La influencia de las particularidades del habla chilota (así como de su cultura) ha tendido por décadas a expandirse tanto hacia el norte como hacia el sur de la Isla Grande, como efecto de la gran movilidad de la gente chilota, motivada fundamentalmente por la pauperización de las tierras y la carencia de suficientes fuentes de trabajo.
} 
dialectales del mapudungun, por lo que ellas pueden ser consideradas como una sola unidad léxica.

De ser así, su existencia no solo revelaría el contacto del campesino de origen hispano con el de origen indígena en una tarea que les es común: el cultivo de la papa, sino que las áreas de troyo y choyo constituirían dos áreas discontinuas periféricas, que en un momento anterior conformaron un área intermedia continua cubierta por una misma unidad léxica incorporada al español, probablemente choyo, con su variante troyo, hoy en franco retroceso. Esta área habría sido fracturada por el neologismo tallo, que se concentra precisamente en las áreas que habría dominado choyo y su variante, dado que la voz canónica brote había reducido considerablemente su presencia (16 ocurrencias en las regiones sur y sur-austral, frente a 32 de tallo) por la mayor penetración en la fase anterior de la palabra de sustrato. Ahora, ipor qué la variante mapuche troyo está en retroceso en su hábitat, a diferencia de lo que ocurre con la variante mapuche-huilliche choyo en el suyo? Es la pregunta que queda pendiente.

No obstante las dificultades ya señaladas para postular isoglosas en el español americano, es posible verificar en otros casos distribuciones geográficas de vocablos bastante marcadas, que obedecen a lo que se puede llamar "patrón regular", y que se deben a motivaciones diferentes de los hablantes, unas más fáciles de explicar que otras. Vayan algunos ejemplos como muestra, tomados nuevamente del ALECh.

El concepto <avispa> (V. mapa 8) puede ilustrar este nuevo patrón, ya que en las regiones centro y sur la respuesta es propiamente avispa, mientras que en la región suraustral se recogió chaqueta amarilla -de manera exclusiva-, expresión metafórica que alude al color amarillo del cuerpo circundado por anillos de color negro de la Vespula germanica, especie carnívora muy agresiva y difícil de controlar. El trazo de una isoglosa separa aquí dos áreas que coinciden con nuestra hipótesis de la frontera lingüística Mehuín-Panguipulli.

Otro caso interesante corresponde a las denominaciones del concepto <sobeo> 'correa con que se ata la lanza o pértigo de la carreta al yugo' (V. mapa 9). En la región o área central, salvo una localidad, se escucha únicamente pertiguero, palabra sin duda asociada por contigüidad a pértigo, es decir, a la lanza de la carreta, voz que se extiende por todo el país donde hay tierras agrícolas, como se puede comprobar en el mapa <lanza $>$, pero de manera exclusiva, es decir, sin competencia léxica, precisamente en la región central, lo que explica perfectamente la distribución de "pertiguero". En la región sur, la única respuesta recogida es cabrehto -metátesis de cabestro, esto es, "correa que se ata al cuello de una caballería para conducirla o atarla con ella'-, que tiene como límite norte la distribución de "pertiguero" (área central), por lo que allí se puede trazar una primera isoglosa ("regular") que coincidiría con nuestra hipótesis de la frontera lingüística constituida por los ríos Itata-Nuble.

En la región sur-austral se ha podido constatar la presencia de otra respuesta, balsón, para denominar al sobeo; sin embargo, en la parte norte de esta región, entra en competencia con "cabrehto", lo que revela que esta última forma se ha extendido más allá del límite de nuestra región sur, es decir, de la línea Mehuín-Panguipulli, de modo que la segunda isoglosa debe trazarse algo más al sur, en el $42^{\circ}$. 
El tercer ejemplo se refiere a <vertedera $>$ 'cada una de las orejeras del arado que sirven para ensanchar el surco' ( $\mathrm{V}$. mapa 10). El nombre técnico vertedera se presenta en tres localidades de la región central, en otras tres de la región sur y solo en dos de la región sur-austral, por lo que parece ser el primer estrato léxico en retroceso evidente, que ha sido desplazado por un segundo estrato: gualeta -alteración vulgar de "aleta" (de animal)-, expresión metafórica que cubre, salvo las excepciones mencionadas, las regiones central y sur y que penetra en la parte norte de la región sur-austral, hasta encontrarse con pala, el segundo neologismo, a la altura del paralelo 42, que se extiende hasta Tierra del Fuego, con Chiloé como muy probable centro irradiador.

De acuerdo con esto, gualeta y pala configuran claramente dos áreas separadas por una frontera que puede ser marcada con una isoglosa que iría algo más al sur que la línea Mehuín-Panguipulli propuesta como hipótesis.

Las experiencias recogidas provenientes de otras latitudes y nuestro análisis del comportamiento de las isoglosas del español de Chile en el material examinado, nos permiten confirmar la existencia del "patrón de piel de leopardo" como formación de isoglosas bastante habitual, pero también se han encontrado unidades de la lengua cuya distribución espacial configura un "patrón de áreas residuales", e incluso, casos de distribuciones bastante marcadas que pueden configurar lo que hemos denominado "patrón regular", por lo que en tanto no se examine una muestra más amplia no cabe afirmar que el primero sea el patrón predominante en la distribución dialectal del habla chilena.

\section{OBRas CITADAS}

ALEC 1982. Atlas Lingüistico-etnográfico de Colombia. 1981-1983. Dir. Luis Flórez. 6 tomos. Bogotá: Instituto Caro y Cuervo.

ALMex. 1990-2003. Atlas Lingüistico de México. Dir. Juan M. Lope Blanch. 6 vols. México: El Colegio de México y F. C. E.

Araya, Guillermo. 1968. Atlas Lingüistico-Etnográfico del Sur de Chile (ALESUCH). Preliminares y Cuestionario. Anejo 1 de Estudios Filológicos. Valdivia: Univ. Austral de Chile.

Catrileo, María. 2010. La lengua mapuche en el siglo XXI. Valdivia: Universidad Austral de Chile.

Chambers, J.K. y Peter Trudgill. 1994. La Dialectología. Trad. Carmen Morán González. Madrid: Visor Libros.

Lenz, Rodolfo. [1987]. Diccionario etimológico de las voces chilenas derivadas de lenguas indígenas americanas. Ed. Mario Ferreccio Podesta. Santiago: Univ. de Chile.

Moreno Fernández, Francisco, ed. 1993. La división dialectal del español de América. Alcalá de Henares: Univ. de Alcalá de Henares.

Pottier, Bernard. 1992. "La variación lingüística y el español de América". Revista de Filología Española LXXII.3-4 (El español de América): 283-296. 
ESTUDIOS FILOLÓGICOS

Wagner, Claudio. 1998. "El Atlas lingüístico y etnográfico de Chile por regiones". EFil 33: 119-129.

---. 2003. "El Atlas lingüístico de México". NRFH LI.1: 193-203.

---. 2016. Atlas lingüístico y etnográfico de Chile por regiones (ALECh). Versión digital en sitio web www.atlaslinguistico.cl .

Winkelmann, Otto. 1996. "La Geolingüística pluridimensional y el análisis de situaciones de contacto lingüístico". Neue Wege der Romanischen Geolinguistik. Acten des Symposiums zur Empirischen Dialecktologie (Heidelberg / Mainz 21-24/10/1991). Kiel: Westensee-Verlag. 342-353. 
ESTUDIOS FILOLÓGICOS

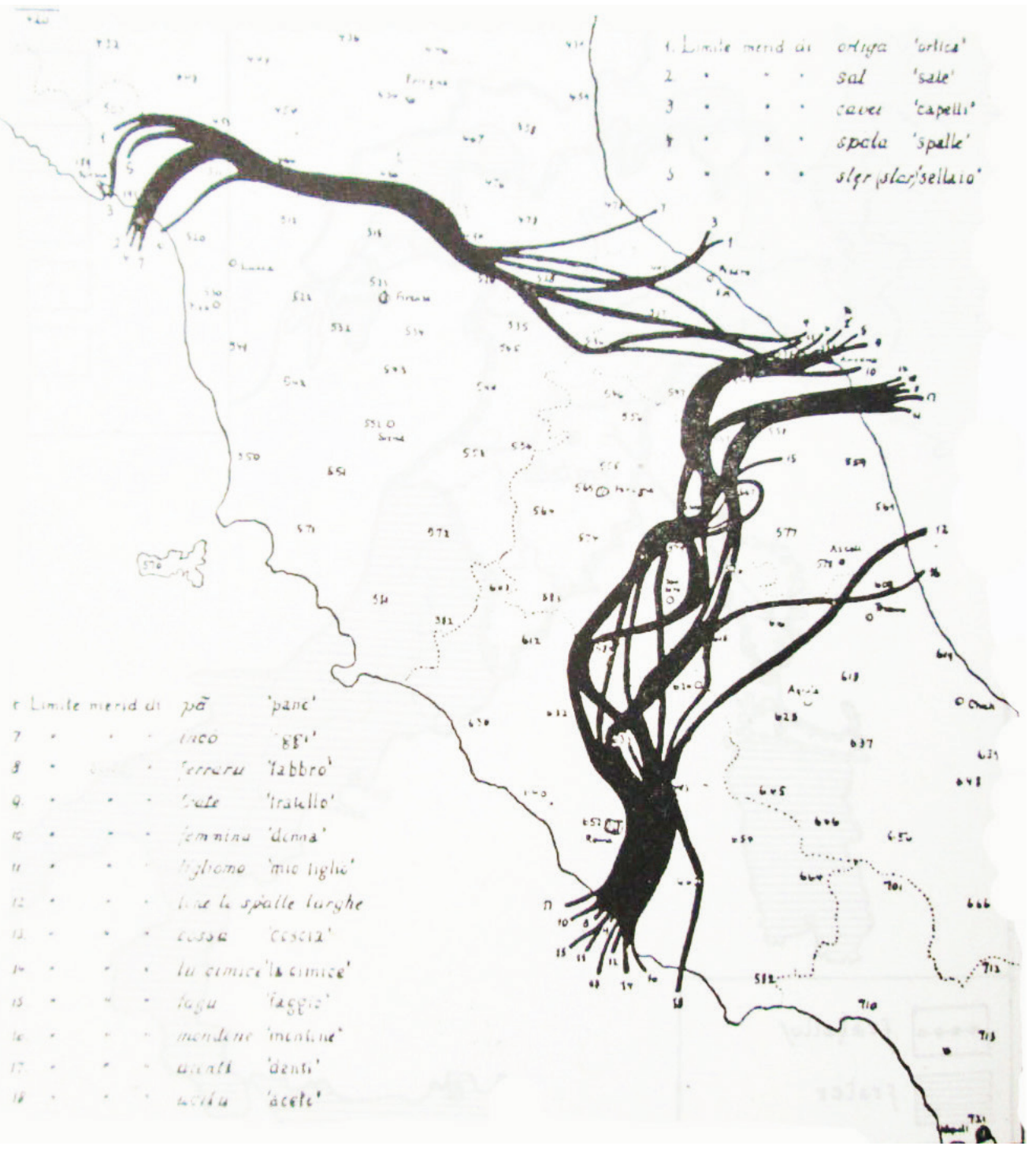

1. Líneas La Spezia - Rimini y Ancona - Cerros Albanos

("Las tres Italias") 


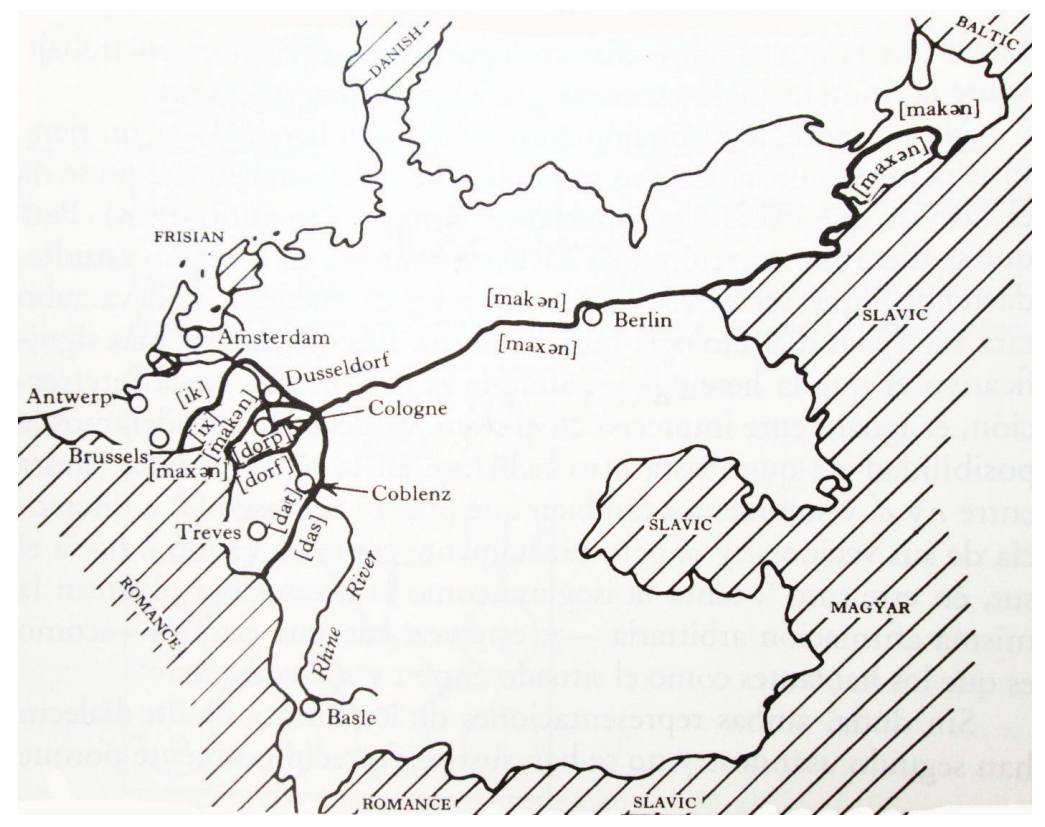

2. Línea de Benrath (Bajo y alto alemán)

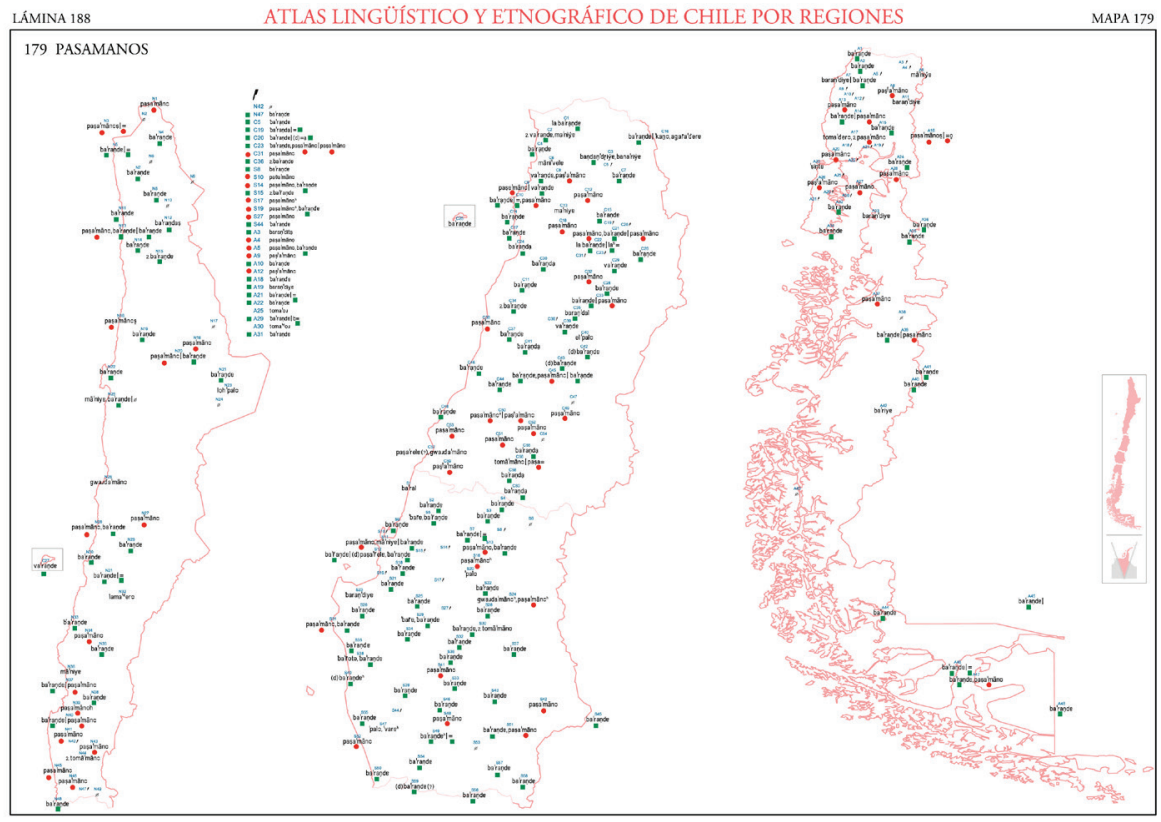

3. Pasamanos 


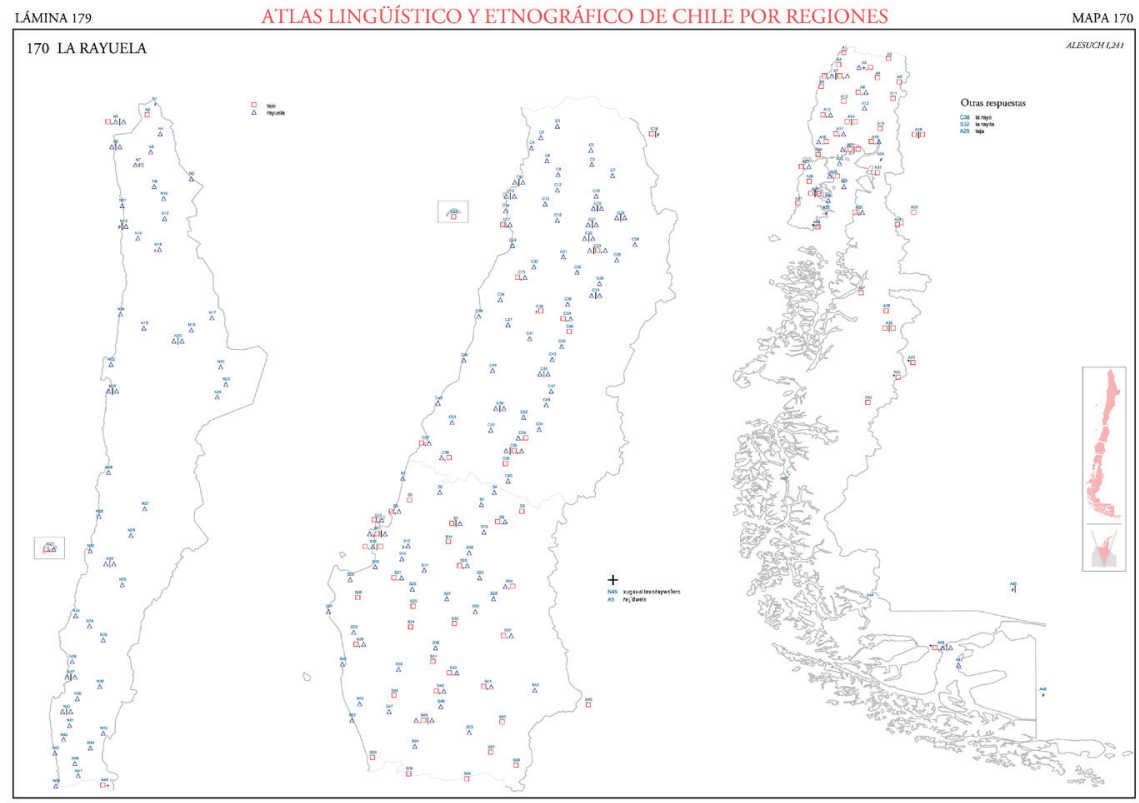

\section{La rayuela}

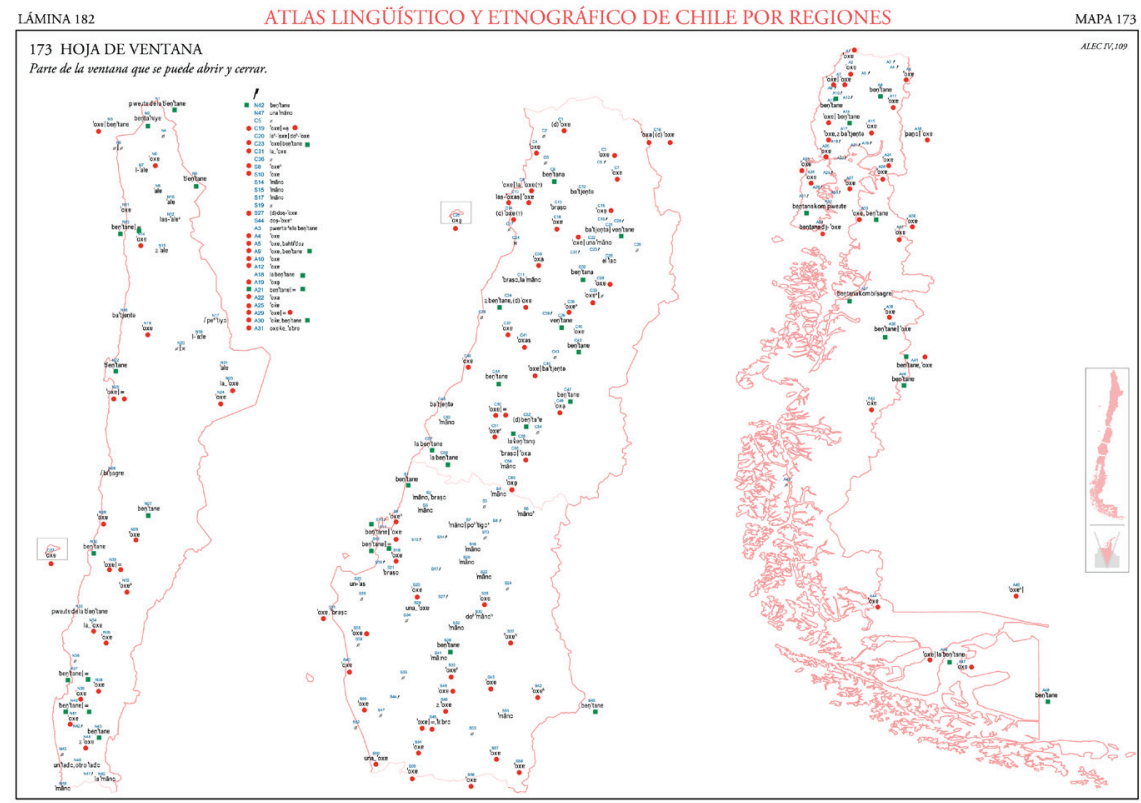

5. Hoja de ventana 


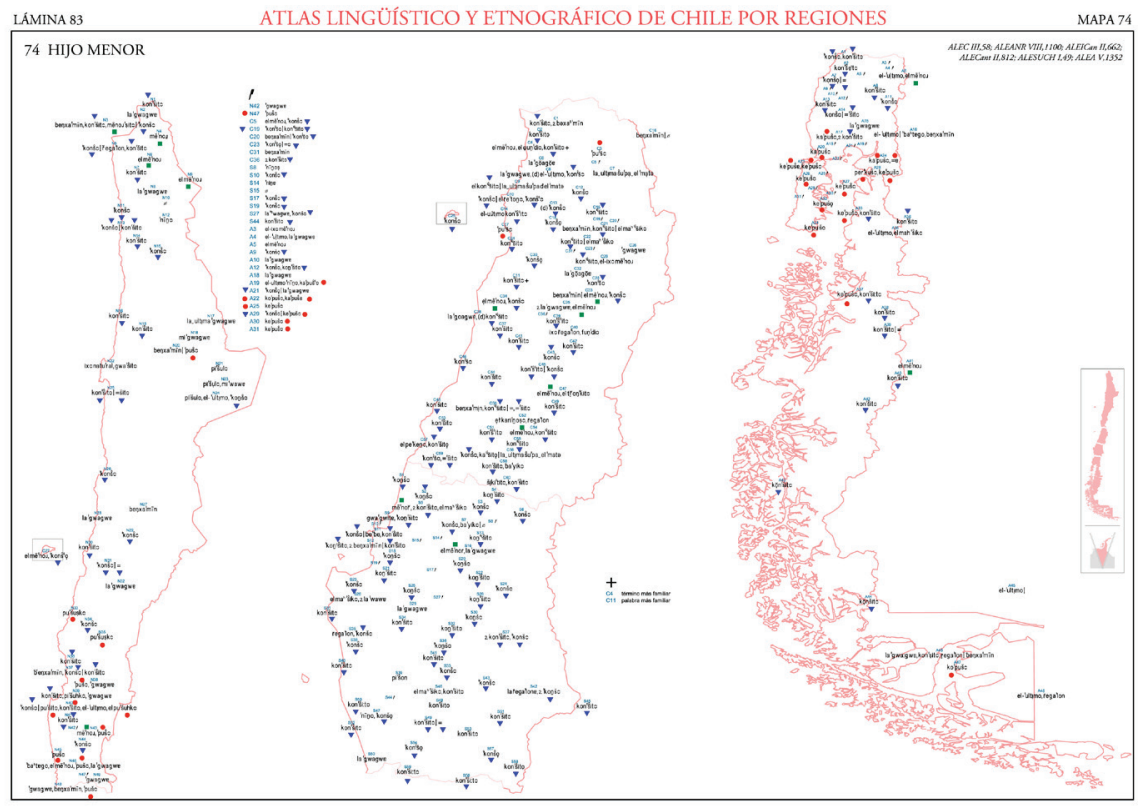

\section{Hijo menor}

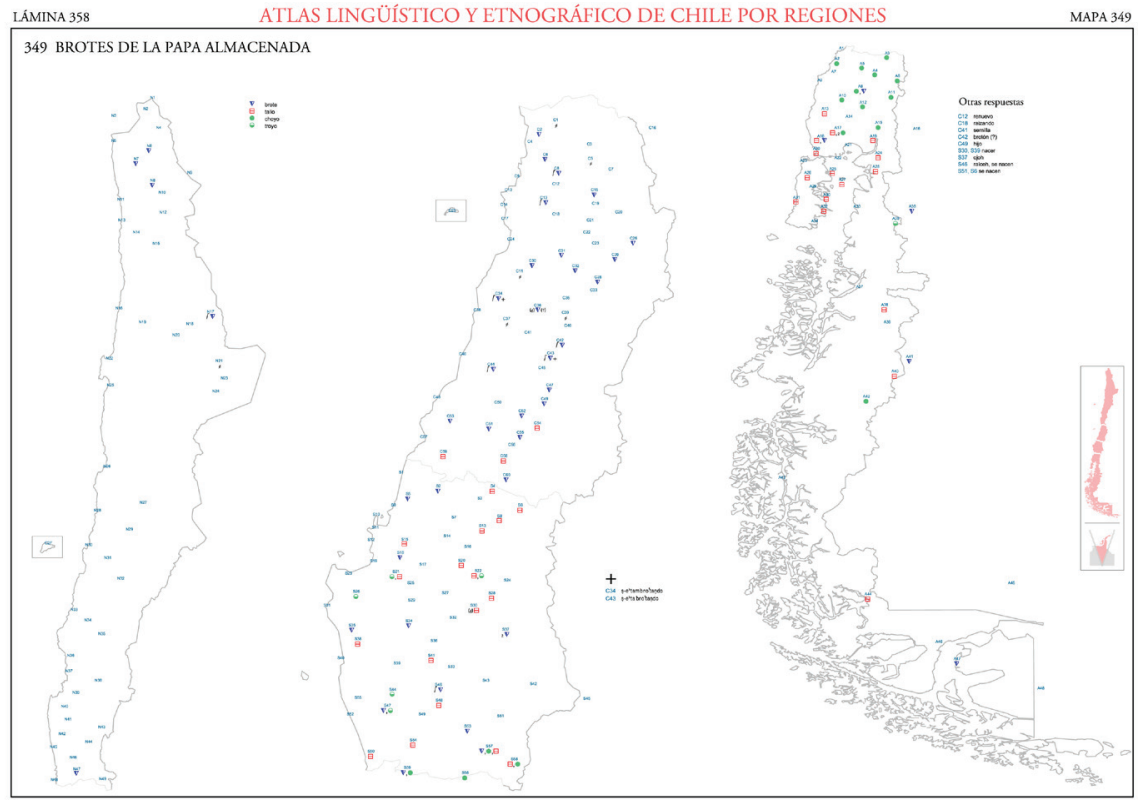

7. Brotes de la papa almacenada 


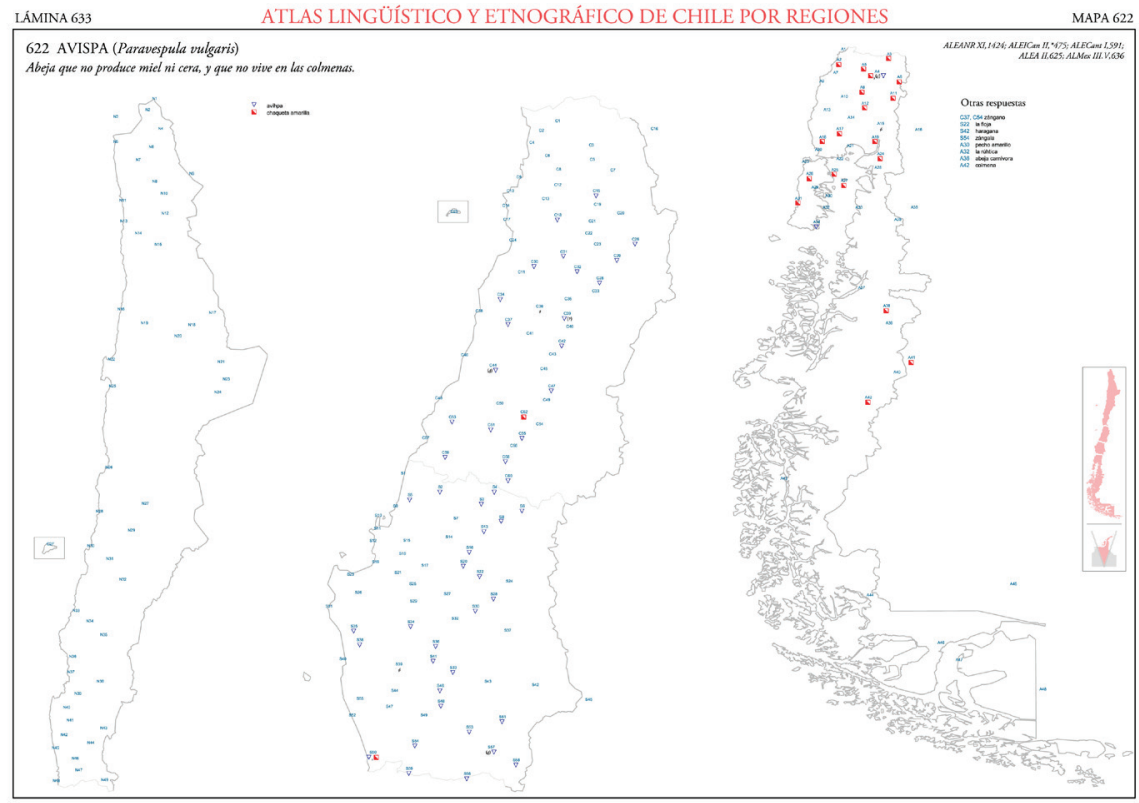

\section{Avispa}

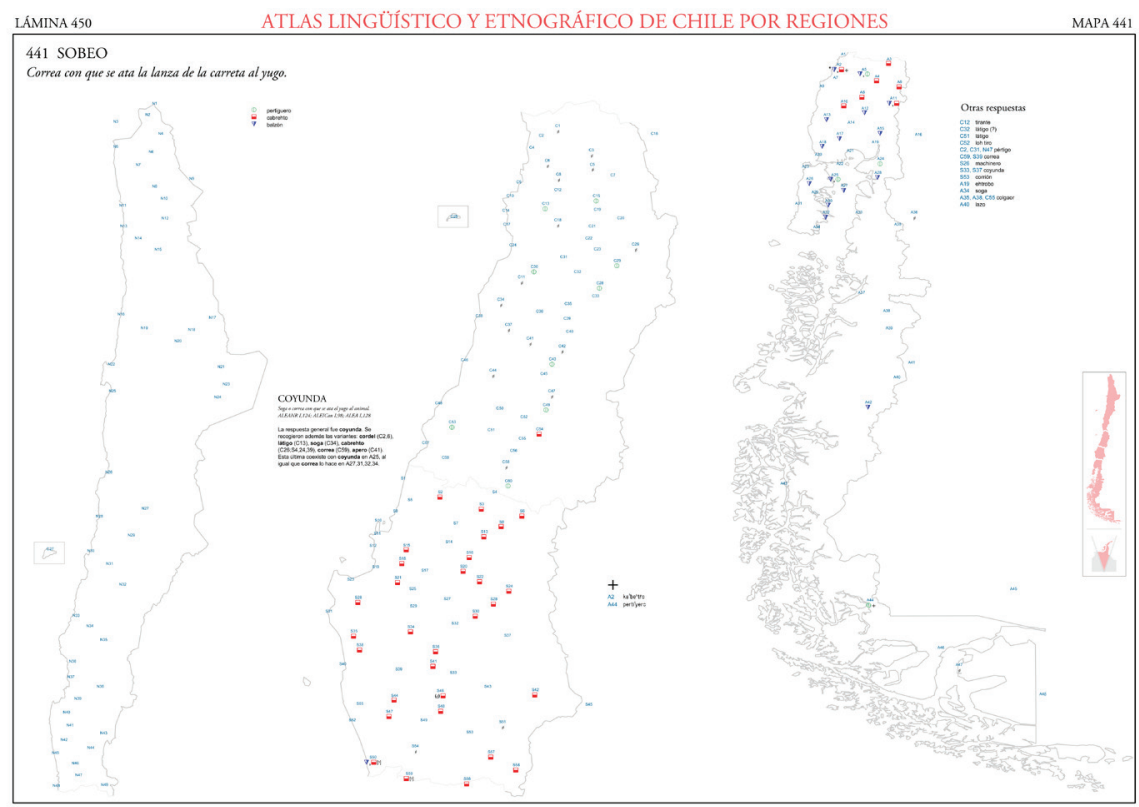

9. Sobeo 
ESTUDIOS FILOLÓGICOS

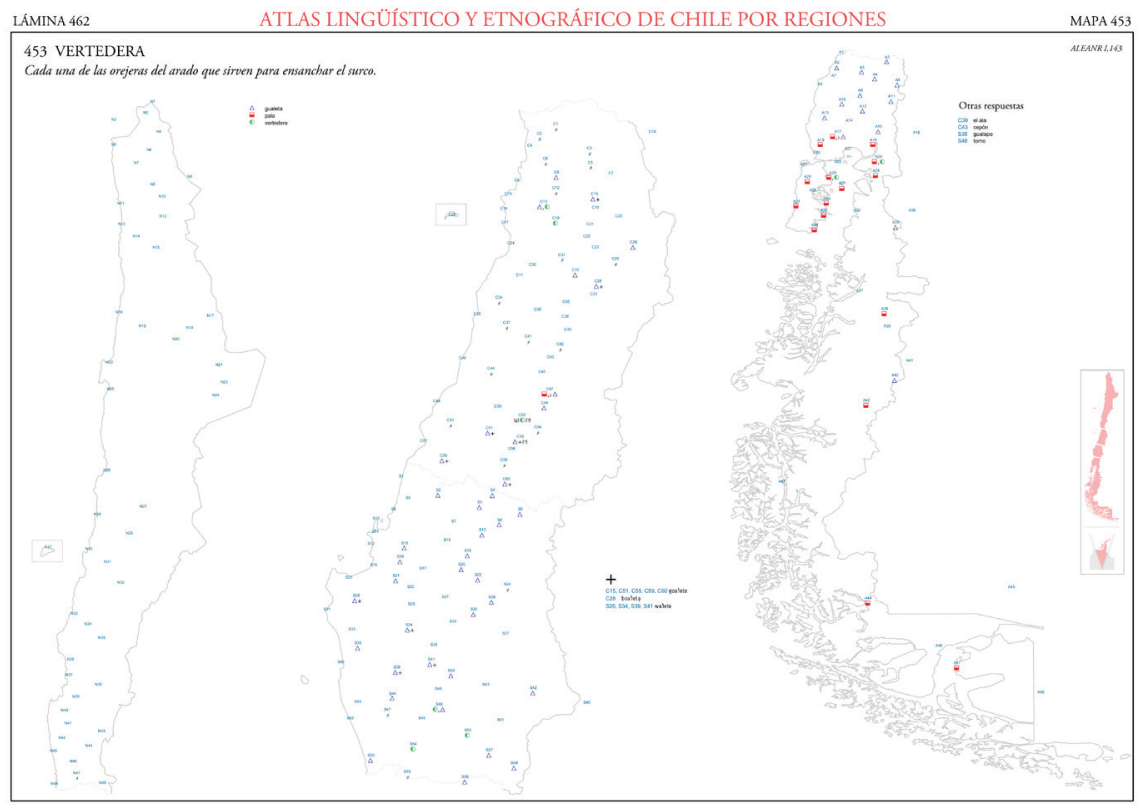

10. Vertedera 